\title{
ESTIMAÇÃO DE FREQÜÊNCIAS POR PREDIÇÃO LINEAR COM DESEMPENHO DE MÁXIMA VEROSSIMILHANÇA
}

\author{
Rodrigo Pinto Lemos \\ Escola de Engenharia Elétrica, \\ Universidade Federal de Goiás - UFG \\ Praça Universitária s/ nº , 74605-220 Goiânia, GO \\ Telefone/Fax: (062)202-0325 \\ lemos@eee.ufg.br
}

\author{
Amauri Lopes \\ Fac. Engenharia_Elétrica e de Computação \\ Universidade Estadual de Campinas - UNICAMP \\ Caixa Postal 6101, 13083-970 Campinas, SP \\ Telefone: (019)788-7502; Fax: (019)788-1395 \\ amauri@decom.fee.unicamp.br
}

Resumo: Este trabalho revisa a aplicação de predição linear para a estimação de frequiências em meios ruidosos, estabelecendo uma formulação unificada. Descreve-se um método, proposto pelos autores, que otimiza a predição linear no sentido dos mínimos quadrados totais, bem como sua solução pelo subespaço de sinal. Seu desempenho é competitivo com aquele de componentes principais. Descrevem-se também métodos recentes que otimizam a predição linear no sentido dos mínimos quadrados totais e que, considerando a estrutura da matriz de dados, alcançam o desempenho de máxima verossimilhança. Demonstra-se a equivalência entre esses métodos e se comparam seus desempenhos.

\begin{abstract}
This work establishes a unifying framework to the use of linear prediction for estimating the unknown frequencies of signals corrupted by additive noise. A method is proposed that optimizes linear prediction in total least squares sense. Also presented is a signal subspace approach to the method. This method is shown to be competitive in relation to that of principal components. In order to make linear prediction perform like maximum likelihood, recent methods take into account the structure imposed by the linear prediction filter on the data matrix. These methods are shown to minimize the same objective function and yield equivalent solutions.
\end{abstract}

Palavras-chave: estimação de freqüências, predição linear, mínimos quadrados totais, máxima verossimilhança.

\section{INTRODUÇÃO}

A estimação de senóides contaminadas por ruído branco tem recebido especial atenção em processamento digital de sinais, por se tratar de um problema clássico com inúmeras aplicações, dentre as quais destacamos radar, sonar, ressonância nuclear magnética, arranjos de antenas [1].

O problema básico consiste em estimar as freqüências das senóides, considerando um número finito de amostras do sinal. Este problema torna-se crítico quando a diferença entre essas freqüências é inferior ao recíproco do intervalo de observação do sinal e à medida em que a relação sinalruído (SNR) diminui. Nestes casos, os métodos tradicionais baseados na transformada de Fourier não oferecem resolução suficiente [2].

O melhor desempenho para tal estimação é alcançado por métodos de Máxima Verossimilhança (ML), porém sob grande esforço computacional. Na tentativa de se obter métodos com desempenho próximo àquele da $\mathrm{ML}$, porém com esforço computacional inferior, Tufts e Kumaresan utilizaram Predição Linear (LP) e o critério dos Mínimos Quadrados (LS), para desenvolver o método FBLP Modificado [3]. Este método realiza a otimização conjunta dos filtros de predição linear progressiva (forward) e regressiva (backward), através do procedimento conhecido como "Forward - Backward Linear Prediction" (FBLP) [2]. Utilizando a decomposição em valores singulares (SVD), define os subespaços de sinal e de ruído para os dados disponiveis e gera uma matriz de dados corrigida, baseada apenas no subespaço de sinal, com conseqüente eliminação da parcela referente ao sub- espaço de ruído. A solução do FBLP Modificado baseia-se nessa matriz, tornando este método computacionalmente mais econômico e com desempenho próximo ao do $\mathrm{ML}$ para valores de SNR não muito pequenos.

Em 1980, Golub e Van Loan [4] introduziram o critério de Mínimos Quadrados Totais (TLS) que otimiza a predição linear de uma forma mais ampla, considerando perturbações não só no sinal desejado, como efetuado no critério LS, mas também no sinal de entrada do preditor [2].

Associando essas duas idéias, Lemos e Lopes [5] propuseram a otimização do método FBLP segundo o critério TLS, originando o método TLS-FBLP. Posteriormente, este método foi modificado pelos autores para utilizar apenas o subespaço de sinal [6] e se demonstrou analiticamente que a solução TLS corresponde na verdade a uma solução LS na qual a matriz de dados e o vetor desejado sofrem restrição conjunta ao subespaço de sinal. O método TLS-FBLP mostrou-se competitivo com o FBLP Modificado, porém seu desempenho, assim como o deste último, ainda é significativamente inferior ao da Máxima Verossimilhança.

Tal comportamento se deve ao fato de que tanto o FBLP Modificado quanto o TLS-FBLP não preservam a estrutura da matriz de dados, inserida pelo filtro de predição linear. Quando se descarta a contribuição do subespaço de ruído, produzindo uma matriz de dados corrigida, descarta-se também sua estrutura original. Embora esta correção proporcione significativa melhora no desempenho da estimação de freqüências, ela desestrutura o esquema de predição linear.

No sentido de conduzir o desempenho da predição linear até o mais próximo daquele obtido com a Máxima Verossimilhança, foram propostos diversos métodos, baseados no critério TLS, que permitem preservar a estrutura da predição ao descartar a contribuição do 


\section{Rodrigo Pinto Lemos e Amauri Lopes \\ Estimação e Freqüências por Predição Linear com Desempenho de Máxima Verossimilhança}

subespaço de ruído. Hua e Sarkar propuseram o método Mínimos Quadrados Totais Branqueado (WTLS) [7]; Abatzoglou e Mendel apresentaram o método Mínimos Quadrados Totais Restrito (CTLS) [8] e De Moor introduziu o método Mínimos Quadrados Estruturado (STLS) [9]. Estes métodos consistem em sofisticações da abordagem TLS, considerando a existência de dependências lineares entre as linhas da matriz de dados estendida. Alternativamente, Bresler e Macovski derivaram uma expressão exata para o critério de Máxima Verossimilhança em termos do polinômio de predição do sinal isento de ruído, originando o método Máxima Verossimilhança Quadrática Iterativa (IQML) [10].

Recentemente, Lemos e Lopes [11] demonstraram que os métodos WTLS, CTLS, STLS e IQML minimizam a norma do mesmo erro e, sob as mesmas restrições, produzem soluções equivalentes.

Este trabalho apresenta uma revisão de todos estes métodos, estabelecendo uma formulação unificada em termos de notação, abordagem e desenvolvimento. Descrevemos detalhadamente os métodos citados e estabelecemos comparações entre suas estruturas e desempenhos. Na seção 2 discutimos as bases da estimação de frequêencias por predição linear, descrevendo o método FBLP Modificado. Na seção 3 introduzimos o método TLS-FBLP e na seção 4 comparamos os desempenhos dos métodos FBLP Modificado e TLS-FBLP. Na seção 5 descrevemos a utilização do critério de Máxima Verossimilhança em estimação de freqüências. Nas seções $6,7,8$ e 9 apresentamos os métodos IQML, CTLS, STLS e WTLS, respectivamente, bem como demonstramos a equivalência entre eles. O desempenho destes métodos equivalentes é apresentado na seção 10 . Por fim, as conclusões gerais zompõem a seção 11 .

\section{MÉTODO FBLP MODIFICADO}

No sentido de obtermos uma formulação mais geral para o problema de estimação de freqüências, utilizaremos exponenciais complexas ao invés de senóides. Sejam $N$ amostras de um sinal $x[n]$, composto pela soma de $M$ exponenciais complexas corrompidas por ruído branco aditivo complexo gaussiano, dado por:

$$
x[n]=\sum_{k=1}^{M} a_{k} \exp \left[j\left(\omega_{k} n+\phi_{k}\right)\right]+y[n], n=0, \ldots, N-1
$$

onde $a_{k}$ são as amplitudes, $\omega_{k}$ são as freqüências angulares $\left(\omega_{k}=2 \pi f_{k}\right.$, e $f_{k}$ é a freqüência normalizada em relação à freqüência de amostragem) e $\phi_{k}$ são as fases das exponenciais. Assumimos que o ruído $y[n]$ tem média nula e partes real e imaginária descorrelacionadas, cada qual com variância $\sigma_{y}^{2} / 2$.

Ao longo do restante deste trabalho estaremos supondo que o número $\mathrm{M}$ de exponenciais complexas é conhecido. A determinação do número $\mathrm{M}$ é um problema importante $\mathrm{e}$ bastante tratado na literatura [2], mas escapa do escopo deste trabalho.

A predição linear permite extrair as frequências do sinal a partir do vetor de coeficientes $w$ ' de um filtro de erro de predição (FEP), otimizado de forma a minimizar o erro quadrático médio de predição [12].

Ulrych e Clayton [12] e Nuttal [13] utilizaram a otimização simultânea de filtros de erro de predição (FEP) forward e backward, segundo o procedimento conhecido como "Forward - Backward Linear Prediction" [2]. Eles demonstraram que este procedimento fornece melhores resultados que a otimização do filtro forward ou do filtro backward isolados.

Aplicando as $N$ amostras de $\mathrm{x}[\mathrm{n}]$ ao filtro de predição forward-backward de ordem $L$, compomos a matriz $\mathbf{A}$ de dados e o vetor $\mathbf{b}$ desejado, associados à predição linear [2], como:

$$
\mathbf{A}=\left[\begin{array}{l}
\mathbf{A}_{f} \\
\hdashline \mathbf{A}_{b}^{-}
\end{array}\right]_{2(N-L) \times L} \text { e } \quad \mathbf{b}=\left[\begin{array}{l}
\mathbf{b}_{f} \\
\hdashline \mathbf{b}_{b}
\end{array}\right]_{2(N-L) \times 1}
$$

tal que os índices "f $f$ " " $b$ " correspondem à predição forward e backward, respectivamente.

As matrizes $\mathbf{A}_{\mathrm{f}}$ e $\mathbf{A}_{b}$ são dadas por:

$$
\begin{aligned}
\mathbf{A}_{f} & =\left[\begin{array}{cccc}
x[L-1] & x[L-2] & \cdots & x[0] \\
x[L] & x[L-1] & \cdots & x[1] \\
\vdots & \vdots & \ddots & \vdots \\
x[N-2] & x[N-3] & \cdots & x[N-L-1]
\end{array}\right] \\
\mathbf{A}_{b}^{*} & =\left[\begin{array}{cccc}
x[1] & x[2] & \cdots & x[L] \\
x[2] & x[3] & \cdots & x[L+1] \\
\vdots & \vdots & \ddots & \vdots \\
x[N-L] & x[N-L+1] & \cdots & x[N-1]
\end{array}\right]
\end{aligned}
$$

onde "*" simboliza o complexo conjugado.

Os vetores $\mathbf{b}_{\mathrm{f}}$ e $\mathbf{b}_{\mathrm{b}}$ são dados por:

$$
\mathbf{b}_{f}=\left[\begin{array}{c}
x[L] \\
x[L+1] \\
\vdots \\
x[N-1]
\end{array}\right] \mathbf{b}_{b}^{*}=\left[\begin{array}{c}
x[0] \\
x[1] \\
\vdots \\
x[N-L-1]
\end{array}\right]
$$

Devido à presença do ruído $\mathrm{y}[\mathrm{n}]$, podemos relacionar $\mathbf{A}$ e $\mathbf{b}$ apenas através de [2]:

$$
A \mathbf{w}^{\prime} \approx \mathrm{b}
$$

onde $\mathbf{w}^{\prime}=\left[w_{1} \ldots w_{L}\right]$ é o vetor de coeficientes de predição.

O vetor w' resultante da otimização forward-backward aplicada a (6) define um polinômio $W(z)$ de ordem $L$ : 


$$
W(z)=-1+w_{1} z^{-1}+w_{2} z^{-2}+\ldots+w_{L} z^{-L}
$$

$\mathrm{Na}$ ausência de ruído, segundo Antunes [14], $W(z)$ tem $M$ de seus zeros sobre a circunferência de raio unitário (CRU), denominados zeros de sinal. Os restantes, denominados zeros estranhos, distribuem-se com alguma uniformidade no interior da CRU, conforme mostrado na Fig. 1 para o caso em que utilizamos $L=24, M=2, N=25$ amostras e as seguintes amplitudes, frequiências e fases:

$$
\begin{array}{lll}
a_{1}=1,0, & \omega_{1}=(1,00) \pi, & \phi_{1}=(-1,00) \pi ; \\
a_{2}=1,0, & \omega_{2}=(1,04) \pi, & \phi_{2}=(-0,79) \pi .
\end{array}
$$

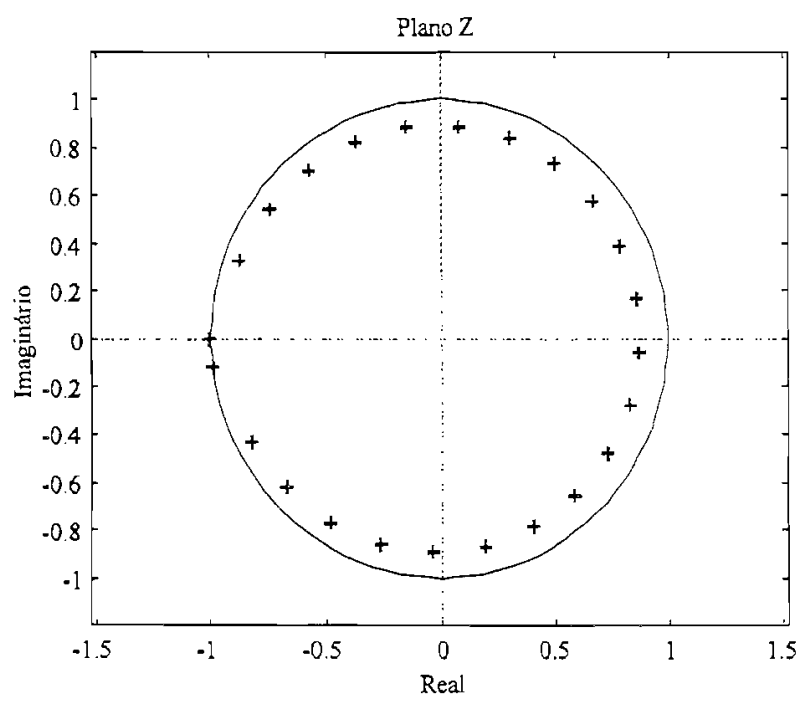

Figura 1. Zeros da função de transferência FBLP, $N=25$ amostras, $\mathrm{L}=24, \mathrm{SNR}=\infty$

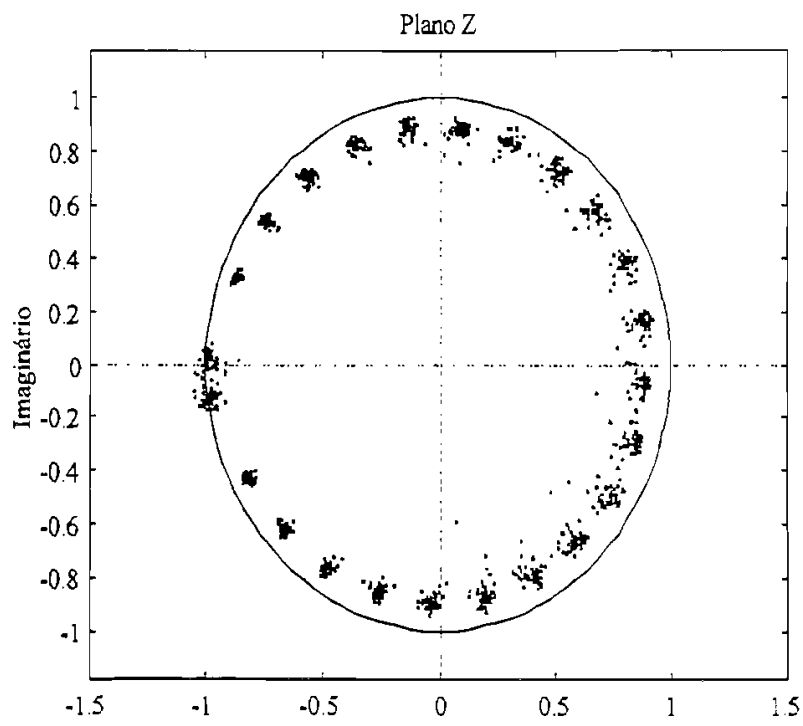

Figura 2. Zeros da função de transferência FBLP, $N=25$, I $=24, \mathrm{SNR}=10 \mathrm{~dB}$. Superposição dos resultados de 50 experimentos.
As posições angulares dos $M$ zeros sobre a CRU determinam as freqüências das exponenciais complexas. Estas são as características básicas do método FBLP para estimação de freqüências.

A contaminação do sinal $x[n]$ pelo ruído aditivo $y[n]$ provoca perturbaçōes sobre os zeros de $W(z)$, produzindo flutuações dos mesmos ao redor de suas posições ideais e degradando o desempenho da estimação. A Fig. 2 evidencia este comportamento, apresentando a superposição dos resultados de 50 experimentos nas mesmas condições da Fig. 1, porém para a relação sinal-ruído, definida como "potência de cada exponencial complexa/ potência do ruído complexo aditivo $y[n] "$ "(SNR), de $10 \mathrm{~dB}$.

Enquanto a SNR apresenta valores da ordem de algumas dezenas de $\mathrm{dBs}$, a degradação de desempenho se deve apenas às flutuações aleatórias dos $M$ zeros de sinal ao redor de suas posições ideais sobre a CRU. Porém, à medida que a SNR decresce, as flutuações dos zeros geram a possibilidade de que algum zero estranho se situe mais próximo da CRU do que os zeros de sinal. Quando isto ocorre, esse zero estranho é interpretado como um zero de sinal e um grande erro de estimação é produzido. $O$ desempenho degrada-se rapidamente, caracterizando um efeito de limiar.

A Fig. 3 permite observar esse comportamento mostrando $o$ inverso da variância do erro de estimação em função da SNR. Estão aí apresentados o limite teórico de desempenho (Limite de Cramér-Rao) e o desempenho do método de Máxima Verossimilhança (ML), para efeito de comparação.

Observamos que as curvas pontilhadas, para SNRs altas, estão muito próximas daquela -associada à ML. Ambas decaem com a redução da SNR até que surge o fenômeno de limiar, com a queda brusca do desempenho.

No sentido de se ter uma igualdade em (6), o critério de Mínimos Quadrados (LS) considera perturbações apenas em b, ou apenas em A, partindo, por exemplo, de:

$$
(\mathbf{A}+\Delta \mathbf{A}) \mathbf{w}^{\prime}=\mathbf{b}
$$

onde $\Delta \mathbf{A}$ tem dimensões $2(N-L) \times L$ e é a matriz de perturbações de $\mathbf{A}$. Assim, a otimização recai sobre uma matriz $\widetilde{\mathbf{A}}=\mathbf{A}+\Delta \mathbf{A}$, que é uma aproximação da matriz $\mathbf{A}$, buscando o vetor $\mathbf{w}^{\prime}$ que minimize a norma de $\Delta \mathbf{A}$ e assegure:

$$
\min \mid \Delta \mathbf{A} \|_{F}, \text { tal que } \mathbf{b} \in \mathcal{J}(\mathbf{A}+\Delta \mathbf{A})
$$

onde $I(\mathbf{A}+\Delta \mathbf{A})$ é o conjunto imagem de $(\mathbf{A}+\Delta \mathbf{A})$. $\mathbf{A}$ solução genérica para este problema é obtida utilizando-se a pseudo-inversa da matriz $\tilde{\mathbf{A}}$ [14]: 


$$
\mathbf{w}^{\prime}=\tilde{\mathbf{A}}^{\#} \mathbf{b}
$$

onde "\#" simboliza a aplicação de pseudo-inversa. Na presença de ruído, a solução w' no sentido LS é obtida simplesmente trocando $\tilde{\mathbf{A}}$ por $\mathbf{A}$ :

$$
\mathbf{w}_{L S}^{\prime}=\mathbf{A}^{\# \mathbf{b}}
$$

A aplicação da Decomposição em Valores Singulares (SVD) à matriz de dados $\mathbf{A}$, permite escrever (11) como:

$$
\mathbf{w}_{L S}^{\prime}=\sum_{k=1}^{\min [2(N-L) L} \frac{1}{f} \sigma_{k} \mathbf{v}_{k} \mathbf{u}_{k}^{H} \mathbf{b},
$$

onde $\sigma_{k}, \mathbf{u}_{k}$ e $\mathbf{v}_{k}$ são os valores e os vetores singulares de $\mathbf{A}$, respectivamente.

No caso sem ruído A tem posto incompleto e igual a $M$, possuindo apenas os $M$ primeiros valores singulares não nulos, correspondentes ao sinal, denominados valores singulares de sinal. No caso ruidoso, A tem posto cheio, possuindo outros $\min [2(N-L), L]-M$ valores singulares não nulos, correspondentes à parcela ruidosa, denominados valores singulares de ruído.

O método FBLP Modificado consiste em desprezar os valores singulares de ruído no cálculo de $\mathbf{w}^{\prime}$, na tentativa de aproximar o caso não ruidoso. Isto eqüivale a fazermos de $\tilde{\mathbf{A}}$ uma aproximação de posto reduzido para $\mathbf{A}$. Considerando que os valores singulares de sinal são os $M$ maiores valores singulares de $\mathbf{A}$, o vetor $\mathbf{w}$ ' preconizado por este método é calculado como:

$$
\mathbf{w}_{\text {Mod. }}^{\prime}=\sum_{k=1}^{M} 1 / \sigma_{k} \mathbf{v}_{k} \mathbf{u}_{k}^{H} \mathbf{b}
$$

Esta estimativa de w' não mais atende ao critério LS, porém sofre menor influência de ruído. Com isto, através da Fig. 4, verificamos que os zeros de $W(z)_{\text {wa. }}$ tendem a se organizar como no caso sem ruído. As curvas contínuas da Fig. 3 permitem observar a redução dos valores de SNR de limiar e o aumento da precisão das estimativas, em relação aos resultados alcançados pelo método FBLP. Podemos observar ainda que os melhores valores de SNR de limiar ocorrem em valores de $\mathrm{L}$ distintos: $\mathrm{L}=8$ para $\circ$ FBLP e L=17 para o FBLP Modificado [3].

\section{MÉTODO TLS-FBLP}

No sentido de obter a igualdade em (6), o critério Mínimos Quadrados Totais (TLS) pressupõe perturbações tanto no vetor desejado $\mathbf{b}$ quanto na matriz de dados $\mathbf{A}$, otimizando w' através de um sistema homogêneo de equações, como no método de Pisarenko [4].

Dessa maneira, descrevemos a predição linear como:

$$
(\mathbf{A}+\Delta \mathbf{A}) \mathbf{w}^{\prime}=(\mathbf{b}+\Delta \mathbf{b})
$$

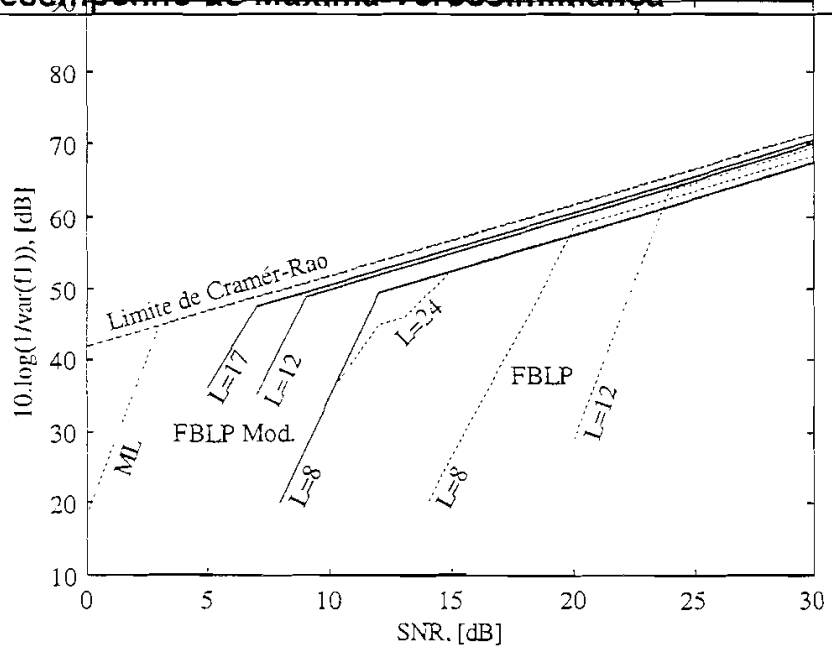

Figura 3. Comparação de desempenho entre os métodos ML, FBLP e FBLP Modificado.

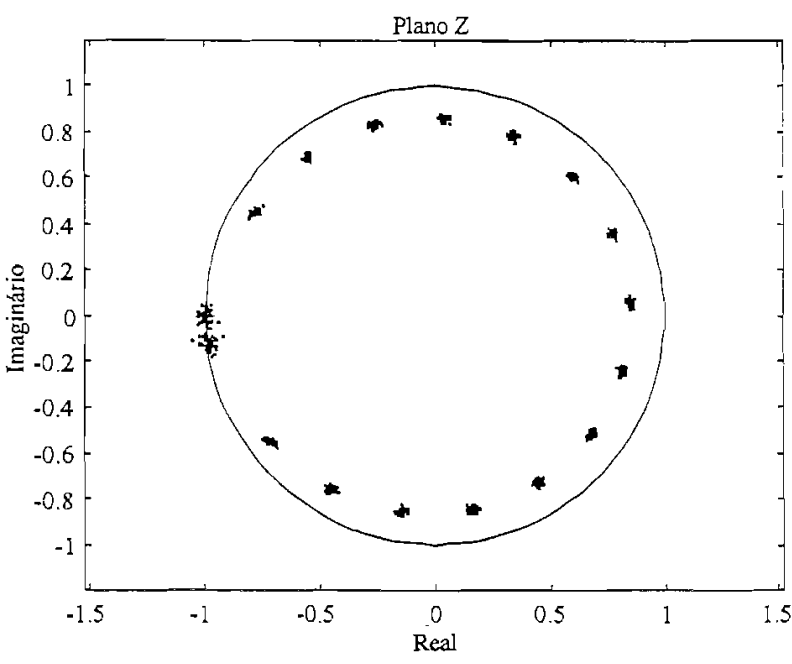

Figura 4. Zeros da função de transferência FBLP Modificado, $N=25$ amostras, $L=17, S N R=10 \mathrm{~dB}$ Superposição dos resultados de 50 experimentos

$$
([\mathbf{b}: \mathbf{A}]+[\Delta \mathbf{b}: \Delta \mathbf{A}]),\left[\begin{array}{l}
-1 \\
\hdashline \mathbf{w}^{\prime}
\end{array}\right]=0
$$

Seja $\mathbf{X}=\left[\begin{array}{l:l}\mathbf{A} & \mathbf{b}\end{array}\right]$ e $\Delta \mathbf{X}=\left[\begin{array}{ll}\Delta \mathbf{b} & \Delta \mathbf{A}]\end{array}\right]$ com dimensões $2(N-L) \times$ $(L+1)$. A solução TLS consiste em encontrar $\mathbf{w}^{\prime}$ que assegure:

$$
\min \|\Delta \mathbf{X}\|_{F}^{2}, \text { tal que }(\mathbf{b}+\Delta \mathbf{b}) \in J(\mathbf{A}+\Delta \mathbf{A})
$$

Seguindo os passos desenvolvidos por Golub e Van Loan [4], a estimativa TLS será obtida a partir da SVD de X:

$$
\mathbf{X}=\left[\begin{array}{l:l}
\mathrm{U}_{X 1} & \mathrm{U}_{X 2}
\end{array}\right]\left[\begin{array}{c:c}
\Sigma_{X 1} & 0 \\
\hdashline \mathbf{0} & \Sigma_{X 2}
\end{array}\right]\left[\begin{array}{c}
\mathbf{V}_{X 1}^{H} \\
\hdashline \mathbf{V}_{X 2}^{H}
\end{array}\right]
$$

Rearranjando (14), obtemos: 
onde:

$\Sigma_{X 1}=\operatorname{diag}\left(\sigma_{X(1)}, \sigma_{X(2)}, \ldots, \sigma_{X(M)}\right)$, com

$$
\sigma_{X(1)} \geq \sigma_{X(2)} \geq \ldots \geq \sigma_{X(M)}>\sigma_{X(M+1)}
$$

$\Sigma_{X 2}=\operatorname{diag}\left(\sigma_{X(M+1)}, \ldots, \sigma_{X(L+1)}\right)=\sigma_{X(L+1)} \mathbf{I}$

$\mathbf{U}_{X 1}=\left\{\mathbf{u}_{X(1)}, \ldots, \mathbf{u}_{X(M)}\right\}$ e $\mathbf{U}_{X 2}=\left\{\mathbf{u}_{X(M+1)}, \cdots, \mathbf{u}_{X(2(N-L))}\right\}$

$\mathbf{v}_{X 1}=\left\{\mathbf{v}_{X(1)}, \ldots, \mathbf{v}_{X(M)}\right\}$ e $\mathbf{v}_{X 2}=\left\{\mathbf{v}_{X(M+1)}, \ldots, \mathbf{v}_{X(L+1)}\right\}$

O mínimo é atingido fazendo-se [4]:

$\Delta \mathbf{X}=-\mathbf{X v v ^ { H }}$

onde $\mathbf{v}$ é qualquer vetor unitário no subespaço $\mathcal{S}_{\mathrm{v}}$ definido pela combinação linear de $\left\{\mathbf{v}_{X(M+1)}, \ldots, \mathbf{v}_{X(L+1)}\right\}$, isto é, dos vetores singulares à direita de $\mathbf{X}$ correspondentes a $\sigma_{X(L+1)}$. A solução TLS pode ser escrita como:

$\mathbf{w}_{T L S}^{\prime}=-\frac{\mathbf{y}}{\alpha}$

na qual o escalar $\alpha$ e o vetor $\mathbf{y}_{L \times 1}$ resultam quando $V_{X 2}$ é submetida uma transformação $\mathbf{H}$ de Householder tal que:

$$
\mathbf{V}_{X 2} \mathbf{H}=\left[\begin{array}{c:c}
\mathbf{0} & \alpha \\
\hdashline \mathbf{Z} & \mathbf{y}
\end{array}\right]
$$

Procedendo à seguinte partição de $V_{X 2}$ :

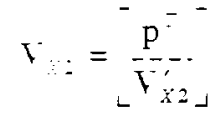

onde $\mathbf{p}^{T}$, com dimensões $1 \times(L+1-M)$, é a primeira linha e $\mathbf{V}_{X 2}^{\prime}$, com dimensões $L \times(L+1-M)$, são as outras linhas de $\mathbf{V}_{X 2}$, concluímos que $\mathbf{H}$ deve satisfazer:

$$
\mathbf{p}^{T} \mathbf{H}=[0, \cdots, 0, \alpha] \text {, ou seja, } \mathbf{H p}^{*}=\left[\begin{array}{c}
0 \\
\vdots \\
0 \\
\alpha^{*}
\end{array}\right]
$$

onde $\mathbf{H}^{H}=\mathbf{H}$. Definindo $\mathbf{e}_{L-M+1}^{T}=[0, \cdots, 0,1]_{1 \times L-M+1}$, a transformação de Householder pode ser escrita como:

$$
\mathbf{H}=\mathbf{I}-2 \frac{s \mathbf{s}^{H}}{\mathbf{s}^{H} \mathbf{s}}
$$

na qual $\mathbf{s}=\mathbf{p}^{*}-\alpha^{*} \mathbf{e}_{L-M+1}$ e $\alpha^{*}$ é calculado como:

$$
\alpha^{*}= \pm \frac{p_{L-M+1}}{\left|p_{L-M+1}\right|}\|\mathbf{p}\|
$$

Para a solução TLS, utilizamos apenas a última coluna de $\mathbf{V}_{X 2} \mathbf{H}=\mathbf{V}_{X_{2}}\left[\mathbf{h}_{1}: \mathbf{h}_{2}: \cdots: \mathbf{h}_{L-M+1}\right]$, dada por:
$\mathbf{V}_{X 2} \mathbf{h}_{L-M+1}=\left[\begin{array}{c}\alpha \\ \hdashline \\ \mathbf{y}\end{array}\right]$

Como $s_{L-M+1}^{*}=p_{L-M+1}-\alpha \quad$ e $\quad \mathbf{s}^{H} \mathbf{s}=2 \alpha^{*}\left(\alpha-p_{L-M+1}\right)$, temos:

$$
\begin{aligned}
\mathbf{h}_{L-M+1} & =\mathbf{e}_{L-M+1}-\frac{2 s_{L-M+1}^{*}}{\mathbf{s}^{H} \mathbf{s}} \mathbf{s} \\
& =\mathbf{e}_{L-M+1}-\frac{2\left(p_{L-M+1}-\alpha\right)}{2 \alpha^{*}\left(\alpha-p_{L-M+1}\right)}\left(\mathbf{p}^{*}-\alpha^{*} \mathbf{e}_{L-M+1}\right) \\
& =\frac{\mathbf{p}^{*}}{\alpha^{*}}
\end{aligned}
$$

Daí obtemos:

$$
\mathbf{y}=\frac{\mathbf{V}_{X 2}^{\prime} \mathbf{p}^{*}}{\alpha^{*}}
$$

e, dado (19), temos que:

$$
\mathbf{w}_{T L S}^{\prime}=-\frac{\mathbf{y}}{\alpha}=-\frac{\mathbf{V}_{X 2}^{\prime} \mathbf{p}^{*}}{\alpha Q^{*}}=-\frac{\mathbf{V}_{X 2}^{\prime} \mathbf{p}^{*}}{\mathbf{p}^{H} \mathbf{p}}
$$

Esta é a solução TLS com base no subespaço de ruído. Podemos encontrar também sua versão com base no subespaço de sinal. Para tanto, fazemos a seguinte partição de $V_{X}$ :

$$
\mathbf{V}_{X}=\left[\begin{array}{l:l}
\mathbf{V}_{X 1} & \mathbf{V}_{X 2}
\end{array}\right]=\left[\begin{array}{c:c}
\mathbf{g}^{T} & \mathbf{p}^{T} \\
\hdashline \mathbf{V}_{X 1}^{\prime} & \mathbf{V}_{X 2}^{\prime}
\end{array}\right]
$$

Enfim, a partir de $\mathbf{V}^{H} \mathbf{V}=\mathbf{I}$, (28) pode ser escrita alternativamente como:

$$
\mathbf{w}_{T L S}^{\prime}=\frac{\mathbf{V}_{X 1}^{\prime} \mathbf{g}^{*}}{1-\mathbf{g}^{H} \mathbf{g}}
$$

que constitui a versão com base no subespaço de sinal para a solução TLS.

As expressões (28) e (30) podem ser aplicadas de forma direta, pois a transformação de Householder está implícita em ambas. Estas duas expressões fornecem a mesma estimativa. Entretanto, conforme as dimensões dos subespaços, aconselha-se o uso daquela que envolver 0 menor número de operações.

\section{COMPARANDO OS DESEMPE- NHOS}

Aqui comparamos os desempenhos dos métodos TLS-FBLP e FBLP Modificado através do cálculo das curvas de desempenho para todas as ordens de predição cabíveis. $O$ procedimento para a obtenção dos pontos do gráfico foi o mesmo adotado na Fig. 3. Entretanto, apresentamos aqui 
superfícies de desempenho (Figs. 6 e 7), as quais permitem acompanhar a variação da performance dos métodos em

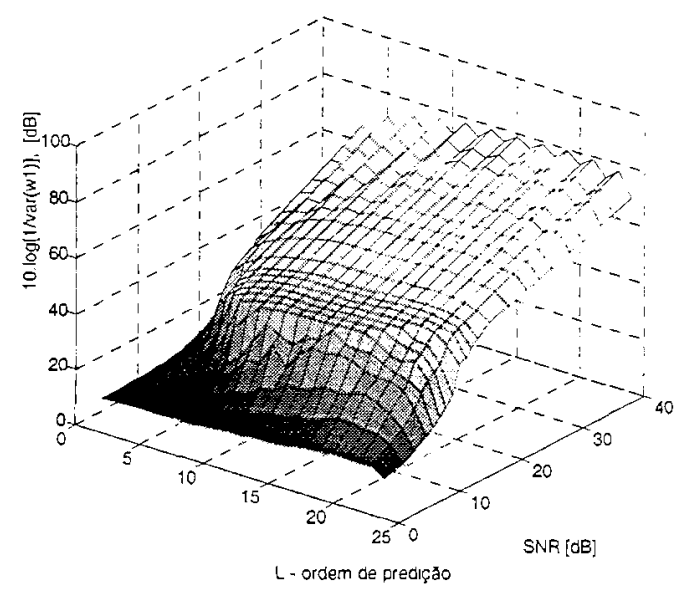

Figura 5. Superfície de desempenho para o método TLS-FBLP (perspectiva à esquerda).

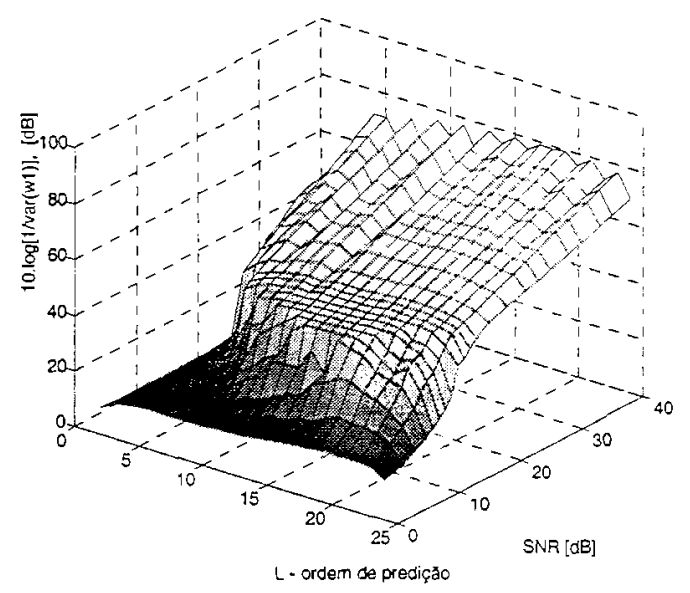

Figura 6. Superfície de desempenho para o método FBLP Modificado (perspectiva à esquerda).

função da ordem de predição e da SNR. Podemos verificar também onde ocorre o melhor desempenho, o pior, ou então acompanhar a variação das SNRs de limiar pelo formato da borda da superfície a partir da qual o desempenho sofre maior degradação.

Através das Figs. 5 e 6 , podemos observar que os desempenhos gerais do TLS-FBLP e do FBLP Modificado, para grandes valores de SNR, oscilam conforme a ordem de predição, sendo melhores para as ordens ímpares e piores para as pares. Entretanto, o FBLP Modificado em ordens pequenas apresenta vales profundos mesmo para grandes SNRs. Já no caso Kumaresan-Prony (KP), $L=24$, tanto o FBLP Modificado quanto o TLS-FBLP, fornecem a mesma curva de desempenho.

Nas Figs. 7 e 8 traçamos também as curvas de nível para as superfícies apresentadas, gerando alternativas para a visualização do contorno de limiar. Nas regiões onde as superfícies são mais ingremes, as linhas ficam mais próximas entre si, definindo o limiar de desempenho e, consequientemente, as respectivas SNRs de limiar para cada ordem de predição.

No TLS-FBLP verificamos que o desempenho acima do limiar varia mais suavemente com a ordem e que o contorno de limiar é mais suave, ou seja, a variação dos valores de SNR de limiar é muito pequena para ordens $L$ de 8 a 18 . Neste caso, o menor valor de SNR de limiar encontrado foi $8 \mathrm{~dB}$ e ocorreu em $L=16$, conforme pode ser observado na Fig. 7.

Observando o FBLP Modificado, podemos constatar que o contorno de limiar é mais agudo, bem como o melhor desempenho ocorre na região de $L=14$ a $L=18$, piorando bastante fora deste intervalo. Portanto, o método TLS-FBLP é mais robusto à variação da ordem de predição $L$ do que o FBLP Modificado, sendo superior a este último para ordens baixas. Pela Fig. 8 percebemos que a menor SNR de limiar para o FBLP Modificado tem o valor de $7 \mathrm{~dB}$ e verifica-se para $L=17$. Este resultado difere do valor $L=18$, obtido por Tufts e Kumaresan, devido ao maior detalhamento das simulações aqui apresentadas.'

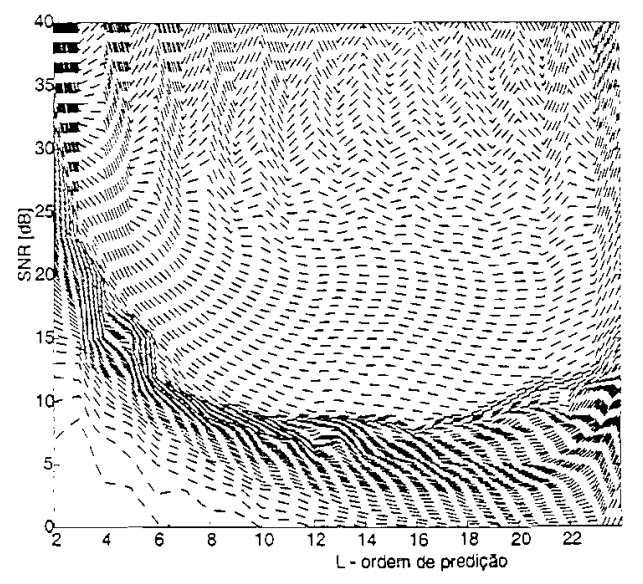

Figura 7. Linhas de nível para a superfície de desempenho TLS-FBLP (vista de topo).

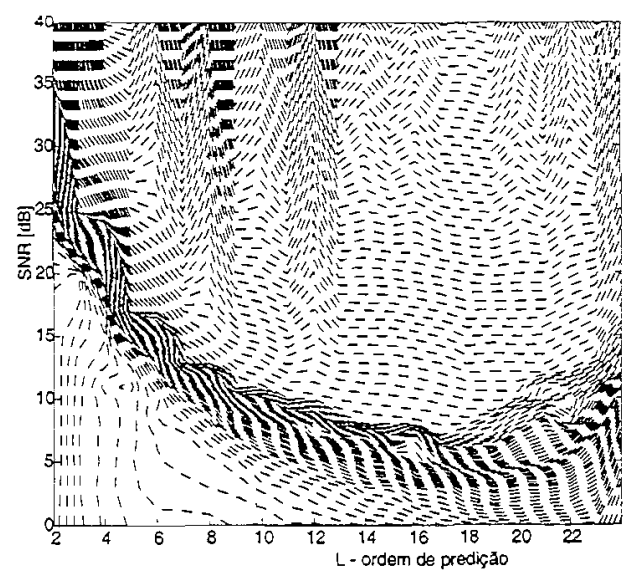

Figura 8. Linhas de nível para a superfície de desempenho FBLP Modificado (vista de topo). 
Os melhores desempenhos tanto para o TLS-FBLP quanto para o FBLP Modificado ocorreram quando, respectivamente, as matrizes de dados $\mathbf{X}$ ou $\mathbf{A}$ tornaram-se aproximadamente quadradas. Assim, a medida empírica de Tufts e Kumaresan [3], para a ordem de menor SNR de limiar do FBLP Modificado, definida como $L=(3 / 4) N$, pode ser substituída pela medida analítica que afirma que $a$ ordem ótima é aquela que faz com que a matriz de dados torne-se o mais próximo da forma quadrada.

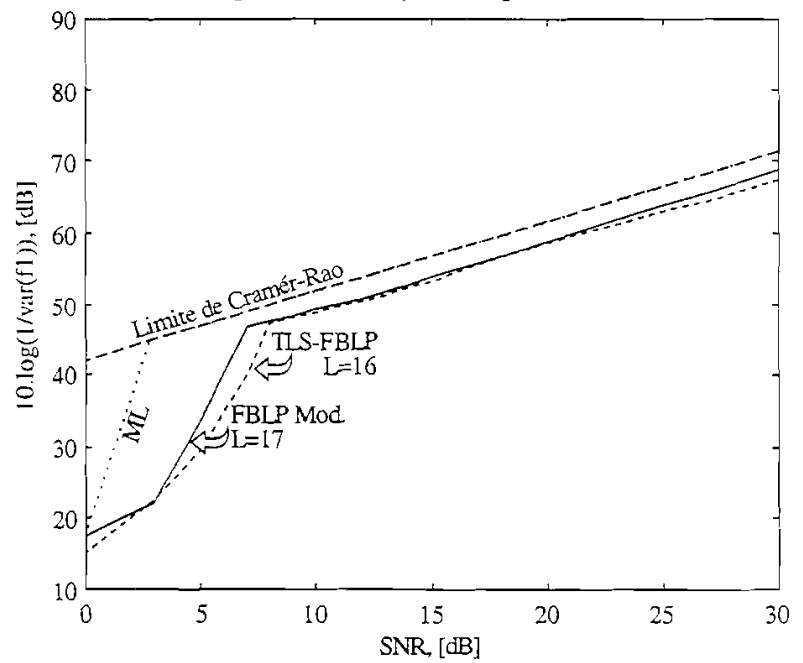

Figura 9. Comparação entre os melhores desempenhos ILS-FBLP e FBLP Modificado.

$\mathrm{Na}$ Fig. 9 destacamos as curvas correspondentes aos menores valores de SNR de limiar para os métodos TLSFBLP e FBLP Modificado, ou seja, as curvas relativas aos melhores desempenhos.

Assim, verificamos que o desempenho do TLS-FBLP revelou-se competitivo com o FBLP Modificado, embora tenham apresentado SNRs de limiar cerca de $4 \mathrm{~dB}$ acima da Máxima Verossimilhança. Isto se deve ao truncamento da SVD, empregado na solução por esses métodos, o qual não preserva a estrutura das respectivas matrizes de dados. Nas próximas seções discutiremos métodos de Máxima Verossimilhança e alguns avanços recentes introduzidos na formulação do critério TLS, de maneira a permitir que levemos em conta a informação de estrutura no processo de otimização, alcançando o desempenho ML.

\section{MÁXIMA VEROSSIMILHANÇA}

Inicialmente vamos recordar a aplicação do critério de Máxima Verossimilhança, preparando o terreno para a formulação do método IQML. Começamos rescrevendo (1) na forma vetorial como:

$$
\mathbf{x}=\mathbf{E} \mathbf{a}_{c}+\mathbf{y}
$$

onde $\mathbf{E}$ é uma matriz de Vandermonde sobre $\exp \left[j \omega_{i} n\right]$ com dimensões $N \times M, \mathbf{a}_{c}$ é um vetor de amplitudes complexas com dimensões $M \times 1$ e y é um vetor com dimensões $N \times 1$ e com partes real e imaginária descorrelacionadas, cada qual com variância $\sigma_{y}^{2} / 2$.
Sob a hipótese de que o ruído é branco e gaussiano, as estimativas $M L$ para as freqüuencias $\omega_{i}$ e para as amplitudes $a_{\mathrm{ci}}, \quad i=1, \ldots, M$, do sinal $x[n]$ são dadas pela solução do seguinte problema de mínimos quadrados não-lineares [15]:

$$
\begin{aligned}
\min _{\mathbf{E} . \mathbf{a}_{c}}\left\|\mathbf{x}-\mathbf{E} \mathbf{a}_{c}\right\|^{2} & =\min _{\mathbf{E}, \mathbf{a}_{c}}\left(\mathbf{x}-\mathbf{E} \mathbf{a}_{c}\right)^{H}\left(\mathbf{x}-\mathbf{E} \mathbf{a}_{c}\right) \\
& =\min _{\mathbf{E}, \mathbf{a}_{c}}\left[\mathbf{x}^{H}\left(\mathbf{x}-\mathbf{E} \mathbf{a}_{c}\right)-\mathbf{a}_{c}^{H} \mathbf{E}^{H}\left(\mathbf{x}-\mathbf{E} \mathbf{a}_{c}\right)\right] \\
& =\min _{\mathbf{E}, \mathbf{a}_{c}}\left[\mathbf{x}^{H}\left(\mathbf{x}-\mathbf{E} \mathbf{a}_{c}\right)-\mathbf{a}_{c}^{H}\left(\mathbf{E}^{H} \mathbf{x}-\mathbf{E}^{H} \mathbf{E} \mathbf{a}_{c}\right)\right]
\end{aligned}
$$

Para qualquer matriz $\mathbf{E}$ dada, a busca de um $\mathbf{a}_{c}$ ótimo correspondente é um problema de mínimos quadrados lineares cuja solução obtém-se por [15]:

$$
\hat{\mathbf{a}}_{c}=\mathrm{E}^{\#} \mathbf{x}=\left(\mathbf{E}^{H} \mathbf{E}\right)^{-1} \mathbf{E}^{H} \mathbf{x}
$$

Podemos substituir (33) em (32), obtendo:

$$
\begin{aligned}
& \min _{\mathbf{E}}\left\|\mathbf{x}-\mathbf{E} \hat{\mathbf{a}}_{c}\right\|^{2}=\min _{\mathbf{E}}\left[\begin{array}{l}
\mathbf{x}^{H}\left(\mathbf{x}-\mathbf{E} \hat{\mathbf{a}}_{c}\right)+ \\
-\hat{\mathbf{a}}_{c}^{H}\left(\mathbf{E}^{H} \mathbf{x}-\mathbf{E}^{H} \mathbf{E}\left(\mathbf{E}^{H} \mathbf{E}\right)^{-1} \mathbf{E}^{H} \mathbf{x}\right)
\end{array}\right] \\
& =\min _{\mathbf{E}}\left[\mathbf{x}^{H}\left(\mathbf{x}-\mathbf{E} \hat{\mathbf{a}}_{c}\right)\right] \\
& =\min _{\mathbf{E}}\left[\mathbf{x}^{H} \mathbf{x}-\mathbf{x}^{H} \mathbf{E}\left(\mathbf{E}^{H} \mathbf{E}\right)^{-1} \mathbf{E}^{H} \mathbf{x}\right]
\end{aligned}
$$

Daí, podemos escrever (32) em duas formulações equivalentes:

$$
\min _{\mathbf{E}}\left(\mathbf{x}-\mathbf{E} \hat{\mathbf{a}}_{c}\right)^{H}\left(\mathbf{x}-\mathbf{E} \hat{\mathbf{a}}_{c}\right)=\min _{\mathbf{E}}\left\{\mathbf{x}^{H}\left[\mathbf{I}-\mathbf{E}\left(\mathbf{E}^{H} \mathbf{E}\right)^{-1} \mathbf{E}^{H}\right] \mathbf{x}\right\}
$$

ou

$$
\min _{\mathbf{E}}\left(\mathbf{x}-\mathbf{E} \hat{\mathbf{a}}_{c}\right)^{H}\left(\mathbf{x}-\mathbf{E} \hat{\mathbf{a}}_{c}\right)=\max _{\mathbf{E}}\left[\mathbf{x}^{H} \mathbf{E}\left(\mathbf{E}^{H} \mathbf{E}\right)^{-1} \mathbf{E}^{H} \mathbf{x}\right](36)
$$

Entretanto, como (35) e (36) são não-lineares nas frequêencias $\omega_{i}$, minimizar (35) ou maximizar (36) exigiria uma busca sobre um espaço $M$-dimensional. Tal busca demanda elevado esforço computacional, o qual pode se tomar proibitivo para grandes valores de $M$.

\section{MÉTODO MÁXIMA VEROSSIMI- LHANÇA QUADRÁTICA ITERATI- VA - IQML}

No sentido de substituir os critérios ML (35) e (36) por expressões mais tratáveis, muitos pesquisadores propuseram transformar o espaço de parâmetros, passando de freqüências para parâmetros $\mathrm{AR}$. Isto é possível observando que, como uma soma de exponenciais complexas pode ser modelada como a resposta ao impulso de um filtro causal ARMA com pólos na circunferência de raio unitário (CRU), podemos equivalentemente determinar as freqüências a partir das posições angulares destes pólos. A localização dos pólos é função dos parâmetros AR. Para compreender esta transformação, escrevemos o polinômio de predição linear como: 
$W(z)=w_{0}+w_{1} z^{-1}+\ldots+w_{L} z^{-1}$ para $M \leq L \leq(N-M)(37)$

$\mathrm{Na}$ ausência de ruído, ele tem raízes sobre a CRU em $z_{i}=\exp \left[j \omega_{i}\right]$, ou seja:

$$
\begin{aligned}
& W\left(\exp \left[j \omega_{i}\right]\right)=\sum_{k=0}^{L} w_{k} \exp \left[-j \omega_{i} k\right]=0 \\
& =\sum_{k=0}^{L} w_{(L-k)} \exp \left[j \omega_{i} k\right]=0^{, i=1, \ldots, M}
\end{aligned}
$$

Então, através da definição da matriz com dimensões $(N-L) \times N$ :

$$
\mathbf{W}^{H}=\left[\begin{array}{cccccccc}
w_{L} & w_{L-1} & \cdots & w_{1} & w_{0} & 0 & \cdots & 0 \\
0 & w_{L} & w_{L-1} & \cdots & w_{1} & w_{0} & \ddots & \vdots \\
\vdots & \ddots & \ddots & \ddots & & \ddots & \ddots & 0 \\
0 & \cdots & 0 & w_{L} & w_{L-1} & \cdots & w_{1} & w_{0}
\end{array}\right]
$$

a partir de (38) podemos escrever:

$$
\mathbf{W}^{H} \mathbf{E}=\mathbf{0}
$$

Da teoria de projeções, temos que [15]:

$$
\mathrm{E}\left(\mathbf{E}^{H} \mathbf{E}\right)^{-1} \mathbf{E}^{H}+\mathbf{W}\left(\mathbf{W}^{H} \mathbf{W}\right)^{-1} \mathbf{W}^{H}=\mathbf{I}
$$

Utilizando (41) em (35) encontramos:

$$
\min _{\mathbf{E}}\left\|\mathbf{x}-\mathbf{E} \hat{\mathbf{a}}_{c}\right\|_{2}^{2}=\min _{\mathbf{W}}\left\{\mathbf{x}^{H} \mathbf{W}\left(\mathbf{W}^{H} \mathbf{W}\right)^{-1} \mathbf{W}^{H} \mathbf{x}\right\}
$$

Ao definirmos a matriz $\mathbf{X}=[\mathbf{b}: \mathbf{A}]$ na forma:

$$
\mathbf{X}=\left[\begin{array}{cccc}
x[L] & x[L-1] & \cdots & x[0] \\
x[L+1] & x[L] & \cdots & x[1] \\
\vdots & \vdots & \ddots & \vdots \\
x[N-1] & x[N-2] & \cdots & x[N-L-1]
\end{array}\right]
$$

e o vetor de coeficientes $\mathbf{w}=\left[\begin{array}{lllll}w_{0} & w_{1} & \ldots & w_{L}\end{array}\right]^{T}$, através da comutatividade da operação de convolução, temos que:

$$
\mathbf{W}^{H} \mathbf{x}=\mathbf{X} \mathbf{w}
$$

Substituindo (44) em (43), finalmente encontramos:

$$
\min _{\mathbf{E}}\left\|\mathbf{x}-\mathbf{E} \hat{\mathbf{a}}_{c}\right\|_{2}^{2}=\min _{\mathbf{w}}\left\{\mathbf{w}^{H} \mathbf{X}^{H}\left(\mathbf{W}^{H} \mathbf{W}\right)^{-1} \mathbf{X} \mathbf{w}\right\}
$$

A expressão (45) constitui-se no erro a ser iterativamente minimizado no método IQML. Esclarecemos que o IQMK, por simplicidade, utiliza $L=M$ e que ao menos a "restrição de não-trivialidade" deve ser aplicada a $\mathbf{w}:\{\mathbf{w}: \mathbf{w} \neq \mathbf{0}\}$. Esta restrição assegura a invertibilidade de $\left(\mathbf{W}^{H} \mathbf{W}\right)$.
Concentrando-nos no método IQMU, além da restrição de não-trivialidade, impõem-se sobre $\mathbf{w}$ as seguintes restriçōes conforme as características dos sinais:

Restrição de estabilidade, para o caso de exponenciais complexas amortecidas: $\{\mathbf{w}$ : $\mathbf{w}$ tem raízes sobre ou dentro da CRU\};

Restrição para o caso de exponenciais complexas nãoamortecidas: $\left\{\mathbf{w}: w_{i}=w_{(L-i)}^{*}, i=0, \ldots, L\right\}$;

Restrição para o caso de senóides reais amortecidas: $\{\mathbf{w}$ : $\operatorname{Im}(\mathbf{w})=0\}$

Restrição para o caso de senóides reais não-amortecidas: $\left\{\mathbf{w}: \operatorname{Im}(\mathbf{w})=0\right.$ e $\left.w_{i}=w_{(L-i)}, i=0, \ldots, L\right\}$.

Devemos observar que a restrição 2 não pode ser atendida se impusermos $w_{0}=-1$. Assim, utilizaremos $\|\mathbf{w}\|=1$ no caso de exponenciais complexas não-amortecidas. A restriçāo 2 , de simetria dos coeficientes do polinômio $W(z)$, pode ser implementada explicitamente sobre $\mathbf{w}$ ou, de maneira implícita, sobre $\mathbf{X}$. A implementação implícita converte o problema atual em outro sem restrições e reduz sua dimensão pela metade. A formulação desta restrição pode seguir por dois caminhos, conforme a paridade de $L$. Aqui apresentamos o caso $L$ par.

Para $L$ par, temos que $L=2 q$. Assim, o vetor $\mathbf{w}$, que tem dimensões $(L+1) \times 1$, possuirá número ímpar de elementos. Portanto, para que a simetria $w_{i}=w_{(L-i)}^{*}, i=0, \ldots, L$ se verifique, é necessário que o elemento central de $\mathbf{w}$ seja real. Daí, particionamos w e $\mathbf{X}$ de forma que:

$$
\mathbf{w}=\left[\begin{array}{lllllll}
w_{0} & \cdots & w_{q-1} & w_{q} & w_{q+1} & \cdots & w_{2 q}
\end{array}\right]^{T}=\left[\begin{array}{lll:l}
\mathbf{w}_{1} & w_{q} & \mathbf{w}_{2}
\end{array}\right]^{T}
$$

$\mathrm{e}$

$$
\mathbf{X} \mathbf{w}=\left[\begin{array}{l:l:}
\mathbf{X}_{1} & \mathbf{x}_{q} \\
\mathbf{X}_{2}
\end{array}\right]\left[\begin{array}{l}
\mathbf{w}_{1} \\
w_{q} \\
\mathbf{w}_{2}
\end{array}\right]=\mathbf{X}_{1} \mathbf{w}_{1}+\mathbf{x}_{q} w_{q}+\mathbf{X}_{2} \mathbf{w}_{2}
$$

Definindo a matriz $\widetilde{\mathbf{I}}$ de reflexão lateral:

$$
\tilde{\mathrm{I}}=\left[\begin{array}{cccc}
0 & \cdots & 0 & 1 \\
0 & \cdots & 1 & 0 \\
\vdots & \ddots & \vdots & \vdots \\
1 & \cdots & 0 & 0
\end{array}\right]
$$

a relação de simetria sobre $\mathbf{w}$ pode ser representada por $\mathbf{w}=\tilde{\mathbf{I}} \mathbf{w}$, ou seja: 


$$
\mathbf{w}_{2}=\widetilde{\mathbf{I}} \mathbf{w}_{1}^{*}
$$

que nos permite escrever:

$$
\begin{aligned}
\mathbf{X} \mathbf{w} & =\mathbf{X}_{1} \mathbf{w}_{1}+\mathbf{x}_{q} w_{q}+\mathbf{X}_{2} \widetilde{\mathbf{I}} \mathbf{w}_{1}^{*} \\
& =\left\{\mathbf{X}_{1}+\mathbf{X}_{2} \widetilde{\mathbf{I}}\right\} \operatorname{Re}\left(\mathbf{w}_{1}\right)+j\left\{\mathbf{X}_{1}-\mathbf{X}_{2} \widetilde{\mathbf{I}}\right\} \operatorname{Im}\left(\mathbf{w}_{1}\right)+\mathbf{x}_{q} w_{q} \\
& =\left[\left(\mathbf{X}_{1}+\mathbf{X}_{2} \widetilde{\mathbf{I}}\right): \mathbf{x}_{q} j j\left(\mathbf{X}_{1}-\mathbf{X}_{2} \tilde{\mathbf{I}}\right)\right]\left[\begin{array}{c}
\operatorname{Re}\left(\mathbf{w}_{1}\right) \\
\hdashline w_{q} \\
\hdashline \operatorname{Im}\left(\mathbf{w}_{1}\right)
\end{array}\right] \\
& =\breve{\mathbf{X}} \mathbf{c}
\end{aligned}
$$

Assim, como $w_{q}$ deve ser real, também o vetor c será real. Portanto, após substituir (50), a minimização (45) sujeita a (49) corresponde a:

$$
\min _{\mathbf{c}}\left\{\mathbf{c}^{T} \breve{\mathbf{X}}^{H}\left(\mathbf{W}^{H} \mathbf{W}\right)^{-1} \breve{\mathbf{X}} \mathbf{c}\right\}
$$

onde devemos impor $c_{0}=1$, para evitar a solução trivial $\mathbf{c}=$ 0 . No sentido de garantir que c seja real, fazemos:

$$
\min _{\mathbf{c}}\left\{\mathbf{c}^{T} \operatorname{Re}\left(\overline{\mathbf{X}}^{H}\left(\mathbf{W}^{H} \mathbf{W}\right)^{-1} \overline{\mathbf{X}}\right) \mathbf{c}\right\}
$$

o que impede que resíduos imaginários do produto matricial danifiquem o cálculo de c.

Agora, definindo a matriz:

$$
\breve{\mathbf{C}}_{X}=\operatorname{Re}\left(\breve{\mathbf{X}}^{H}\left(\mathbf{W}^{H} \mathbf{W}\right)^{-1} \overline{\mathbf{X}}\right)
$$

podemos escrever (52) na forma de uma expressāo de solução iterativa como:

$$
\min _{\mathbf{c}^{(k+1)}}\left\{\left(\mathbf{c}^{(k+1)}\right)^{T} \breve{\mathbf{C}}_{X}^{(k)} \mathbf{c}^{(k+1)}\right\}
$$

onde $c_{0}^{(k+1)}=1$. Propomos transformar (54) em um problema TLS, pois fazendo sua derivada igual a zero obtemos:

$$
\overline{\mathbf{C}}_{X}^{(k)} \mathbf{c}^{(k+1)}=\mathbf{0}
$$

cuja solução pode ser obtida segundo o critério TLS. Assim, a partir da SVD de $\check{\mathbf{C}}_{X}^{(k)}$, dada por:

$$
\overline{\mathrm{C}}_{X}^{(k)}=\mathrm{U}_{C x}^{(k)} \Sigma_{C x}^{(k)}\left(\mathrm{V}_{C x}^{(k)}\right)^{H}
$$

calculamos $\mathrm{c}^{(k+1)}$ através de [4]:

$$
\mathbf{c}^{(k+1)}=-\frac{\mathbf{v}_{C x(L+1)}^{(k)}}{v_{C x(L+1,1)}^{(k)}}
$$

onde $\mathbf{v}_{C x(L+1)}^{(k)}$ é a última coluna de $\mathbf{V}_{C x}^{(k)}$ e $v_{C x(L+1,1)}^{(k)}$ O primeiro elemento de $\mathrm{v}_{C x(L+1)}^{(k)}$. Obtido $\mathrm{c}^{(k+1)}$, reconstruímos $\mathbf{w}_{1}$ e depois w. Os procedimentos (50) a (57) são repetidos até a convergência.
Portanto, no caso de exponenciais complexas puras, devemos impor a restrição 2 , o que foi conseguido através dos passos (50) a (57)

Outro ponto que devemos observar é que, embora a matriz $\mathbf{W}$ tenha posto completo, tanto ela quanto $\left(\mathbf{W}^{H} \mathbf{W}\right)$ são mal-condicionadas por construção. Isto pode levar a imprecisões no cálculo da inversa $\left(\mathbf{W}^{H} \mathbf{W}\right)^{-1}$, prejudicando o cálculo de $\breve{\mathbf{C}}_{X}^{(k)}$ e, conseqüentemente, o de w. A seguir, descrevemos o método de Clark e Scharf [16] que nos permite obter $\left(\mathbf{W}^{H} \mathbf{W}\right)^{-1}$ sem calcular diretamente a inversa.

Primeiramente, definimos a matriz:

$$
\mathrm{T}^{H}=\left[\begin{array}{cccccccc}
w_{0} & 0 & \cdots & 0 & w_{L} & \cdots & w_{2} & w_{1} \\
w_{1} & w_{0} & 0 & \cdots & 0 & w_{L} & \cdots & w_{2} \\
\vdots & \ddots & \ddots & \ddots & & \ddots & \ddots & \vdots \\
w_{L-1} & \cdots & w_{1} & w_{0} & 0 & \cdots & 0 & w_{L}
\end{array}\right]
$$

tal que podemos construir a matriz circulante com dimensōes $N \times N$ :

$$
\mathbf{G}^{H}=\left[\frac{\mathbf{T}^{H}}{\mathbf{W}^{H}}\right]
$$

Isto nos permite definir ainda a seguinte matriz circulante:

$$
\mathbf{Q}=\mathbf{G}^{H} \mathbf{G}=\left[\begin{array}{c:c}
\mathbf{T}^{H} \mathbf{T} & \mathbf{T}^{H} \mathbf{W} \\
\hdashline \mathbf{W}^{H} \mathbf{T} & \mathbf{W}^{H} \mathbf{W}
\end{array}\right]
$$

Decompondo $\mathbf{Q}$ em autovalores é autovetores:

$$
\mathbf{Q}=\mathrm{U}_{Q} \Lambda_{Q} \mathbf{U}_{Q}^{H}
$$

podemos calcular sua inversa facilmente através de:

$$
\mathbf{Q}^{-1}=\mathbf{U}_{Q} \Lambda_{Q}^{-1} \mathbf{U}_{Q}^{H}
$$

Agora, particionamos $\mathrm{Q}^{-1}$ como:

$$
\mathrm{Q}^{-1}=\left[\begin{array}{c:c}
\mathrm{r} & \mathrm{s} \\
\hdashline \mathrm{s}^{H} & \mathrm{t}
\end{array}\right]
$$

onde $\mathbf{r}$, s e $\mathbf{t}$ têm dimensões $L \times L, L \times(N-L)$ e $(N-L)$ $\times(N-L)$, respectivamente. Como $\mathbf{Q}^{-1}$ é também uma matriz circulante, sua inversa, ou seja, $\mathbf{Q}$, pode ser escrita como:

$$
\mathbf{Q}=\left[\begin{array}{c:c}
\ddots & \vdots \\
\hdashline \cdots & \left(\mathbf{t}-\mathbf{s}^{H} \mathbf{r}^{-1} \mathbf{s}\right)^{-1}
\end{array}\right]
$$

Aqui mostramos somente o bloco inferior direito, pois é o de interesse na solução. Comparando (64) a (60), finalmente podemos concluir que:

$$
\left(\mathbf{W}^{H} \mathbf{W}\right)^{-1}=\mathbf{t}-\mathbf{s}^{H} \mathbf{r}^{-1} \mathbf{s}
$$


$\therefore$ ¿ssim, esta expressão garante maior estabilidade ao método -2MI, pois permite calcular a inversa de $\left(\mathbf{W}^{H} \mathbf{W}\right)$ sem - zzê-lo diretamente. Além disso, ela também permite reduzir : gnificativamente a complexidade computacional, pois a =-ior inversa exigida passa a ser a de $r$, que é Toeplitz e :m dimensões $L \times L$.

\section{MÉTODO MÍNIMOS QUADRADOS TOTAIS RESTRITO - CTLS}

-A partir da equação de predição linear para o caso ruidoso:

$$
\mathbf{X} \mathbf{w}=\mathbf{0}
$$

onde $\mathbf{X}=[\mathbf{b}: \mathbf{A}]$, a otimização TLS de $\mathbf{w}$ leva em conta perturbações tanto em A quanto em b. Assim, escrevemos:

$$
(\mathbf{X}+\Delta \mathbf{X}) \mathbf{w}=\mathbf{0}
$$

A solução TLS clássica otimiza w sujeito a (67), pela minimização do produto $\Delta \mathbf{X w}$ sem qualquer consideração adicional. O método CTLS surge como uma melhoria do TLS, por levar em conta a estrutura da matriz de dados estendida X na otimização, ou seja, se ela é Toeplitz (FLP), Hankel (BLP), Hankel-Toeplitz (FBLP), etc. Isto é especialmente interessante quando as entradas ruidosas da matriz de dados são linearmente dependentes. Neste caso, a solução TLS clássica pode não produzir estimadores estatísticos ótimos.

Como uma perturbação em uma amostra qualquer passa pelo filtro de predição juntamente com a própria amostra, $\Delta \mathbf{X}$ é estruturada da mesma forma que $\mathbf{X}$. No sentido de se obter estimativas mais precisas, a informação de estrutura dos componentes do ruído é fatorada em um vetor de perturbações $\Delta \mathbf{x}$ e em um conjunto de matrizes $\left\{\mathbf{F}_{i}\right\}$ de posto incompleto e com a mesma estrutura da matriz de Eados estendida original. Esta restriçāo define o método ILS Restrito (CTLS) [8]:

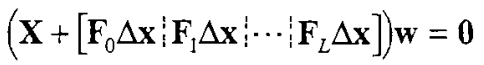

onde $\Delta \mathbf{x}$ é formado pelas $K$ amostras de ruído suficientes para compor $\Delta \mathbf{X}$ (se $\Delta \mathbf{x}$ não for branco, faz-se seu branqueamento). Assim, escrevemos (68) como:

$$
\mathbf{X} \mathbf{w}+\left(\sum_{i=0}^{L} \mathbf{F}_{i} w_{i}\right) \Delta \mathbf{x}=\mathbf{0}
$$

Podemos verificar que $\mathbf{W}^{H}=\sum_{i=0}^{L} \mathbf{F}_{i} w_{i}$, [11]. Logo:

$$
\mathbf{X} \mathbf{w}+\mathbf{W}^{H} \Delta \mathbf{x}=\mathbf{0}
$$

Assim, minimizar $\|\Delta \mathbf{X}\|_{F}$ sujeito a (67) é equivalente a minimizar $\|\Delta \mathbf{x}\|_{2}$ sujeito a (70). Então, $\Delta \mathbf{x}$ é dado por:

$$
\Delta \mathbf{x}=-\left(\mathbf{W}^{H}\right)^{\ddagger} \mathbf{X} \mathbf{w}
$$

A norma mínima de $\Delta \mathbf{x}$ é obtida fazendo-se:

$\min \|\Delta \mathbf{x}\|_{2}^{2}=\min \left(\Delta \mathbf{x}^{H} \Delta \mathbf{x}\right)=\min _{\mathbf{w}}\left[\mathbf{w}^{H} \mathbf{X}^{H} \mathbf{W}^{\#}\left(\mathbf{W}^{\ddot{H}}\right)^{H} \mathbf{X} \mathbf{w}\right]$

Se $\mathbf{W}^{H}$ tem posto completo, então podemos escrever (72) como:

$$
\min \|\Delta \mathbf{x}\|_{2}^{2}=\min _{\mathbf{w}}\left\{\mathbf{w}^{H} \mathbf{X}^{H}\left(\mathbf{W}^{H} \mathbf{W}\right)^{-1} \mathbf{X} \mathbf{w}\right\}
$$

Portanto a solução CTLS é dada pelo vetor $\mathbf{w}$ que minimiza (73). Devemos observar que (73) tem a mesma forma que (45), exceto que na formulação TLS normalmente se impõe $w_{0}=-1$, enquanto que no IQML impõe-se $\|\mathbf{w}\|=1$. Pelo lema a seguir mostramos que isso não afeta a minimização [17]:

Lema 1: Seja $\mathbf{A} \in \mathbb{C}^{g \times h}, \mathbf{z} \in \mathbb{C}^{h \times 1}, \mathbf{Q}_{z} \in \mathbb{C}^{g \times z}$ onde os elementos de $\mathbf{Q}_{z}$ são funções quadráticas dos componentes de z. Seja f uma função multivariável de $\mathrm{z}$ : $\mathrm{f}(\mathbf{z})=\mathbf{z}^{H} \mathbf{A}^{H} \mathbf{Q}_{z}^{-1} \mathbf{A} \mathbf{z}$. Portanto $\mathrm{f}(\alpha \mathbf{z})=\mathrm{f}(\mathbf{z}), \forall \alpha \neq 0$.

Prova: A partir da definição da função $f$, temos que $f(\alpha z)=(\alpha z)^{H} \mathbf{A}^{H} \mathbf{Q}_{\alpha z}^{-1} \mathbf{A}(\alpha z)$. Como os elementos de $\mathbf{Q}_{\alpha z}$ são funções quadráticas dos componentes de $\alpha \mathbf{z}$, temos que $\mathbf{Q}_{\alpha z}=\left(\alpha^{*} \alpha\right) \mathbf{Q}_{z}$. Assim, substituindo $\mathbf{Q}_{\alpha z}$ em $\mathrm{f}(\alpha \mathbf{z})$, temos:

$\mathrm{f}(\alpha \mathbf{z})=\alpha^{*} \mathbf{z}^{H} \mathbf{A}^{H}\left(\alpha^{*} \alpha\right)^{-1} \mathbf{Q}_{z}^{-1} \mathbf{A} \alpha \mathbf{z}=\mathbf{z}^{H} \mathbf{A}^{H} \mathbf{Q}_{z}^{-1} \mathbf{A} \mathbf{z}=\mathrm{f}(\mathbf{z}), \forall \alpha \neq 0$.

Logo, existe um escalar $\alpha=-w_{0}^{-1}$ que pondera $w$ tio sentido de se obter $\alpha w_{0}=-1$, sem afetar a minimização conseguida. Isto significa que se atinge os minimes de 7731 e de (45) com um mesmo vetor $\mathbf{w}$ de coeficientes. Assim, podemos afirmar que (73) é igual a (45) e que, portanto, CTLS e IQML minimizam o mesmo erro, produzindo a mesma solução.

\section{MÉTODO MÍNIMOS QUADRADOS TOTAIS ESTRUTURADO - STLS}

Como dito antes, o critério TLS não produz estimadores estatisticamente ótimos quando as entradas ruidosas da matriz de dados estendida sāo linearmente dependentes, ou seja, quando ela é estruturada. Para que a estrutura de $(\mathbf{X}+\Delta \mathbf{X})$ seja a mesma de $\mathbf{X}$, o método CTLS impõe esta estrutura sobre $\Delta \mathbf{X}$. Já o método Mínimos Quadrados Totais Estruturado (STLS) impõe a estrutura diretamente sobre $(\mathbf{X}+\Delta \mathbf{X})$.

Definindo $\tilde{\mathbf{X}}=(\mathbf{X}+\Delta \mathbf{X})$ e $\tilde{\mathbf{x}}=\mathbf{x}+\Delta \mathbf{x}$, a partir da escolha de um conjunto adequado de matrizes fixas $\mathbf{X}_{i}$ 
$\in \mathbb{C}^{(N-L) \times(L+1)}, \quad i=0, \ldots, K-1$, compostas por " 0 "e " 1 ", podemos descrever a informação de estrutura de $\mathbf{X}$ através de:

$$
\mathbf{X}=\sum_{i=0}^{K-1} x_{i} \mathbf{X}_{i}+\mathbf{X}_{K}
$$

e impomos esta mesma estrutura sobre a matriz $\tilde{\mathbf{X}}$ decompondo-a no mesmo conjunto de matrizes:

$$
\widetilde{\mathbf{X}}=\sum_{i=0}^{K-1} \tilde{x}_{i} \mathbf{X}_{i}+\mathbf{X}_{K}
$$

Normalmente, $K=N$, ou seja, consideramos todas as $N$ amostras disponíveis ao construirmos $\mathbf{X}$ e $\tilde{\mathbf{X}}$. Podemos agora escrever o problema STLS como:

$\min _{\tilde{3} . *} \sum_{i=0}^{K-1}\left(x_{i}-\tilde{x}_{i}\right)^{*}\left(x_{i}-\tilde{x}_{i}\right)$ tal que $\tilde{\mathbf{X}} \mathbf{w}=0, \mathbf{w}^{H} \mathbf{w}=1$

sujeito a (74) e (75).

No sentido de solucionar o problema de minimização, tomamos o Lagrangiano de (76) como em [9]:

$\mathcal{L}(\tilde{\mathbf{x}}, \mathbf{w}, \mathbf{l}, \lambda)=\sum_{i=0}^{N-1}\left(x_{i}-\tilde{x}_{i}\right)^{*}\left(x_{i}-\tilde{x}_{i}\right)-\mathbf{l}^{H} \tilde{\mathbf{X}} \mathbf{w}+\lambda\left(1-\mathbf{w}^{H} \mathbf{w}\right)(77)$

onde $\mathbf{l} \in \mathbb{C}^{(N-L) \times 1}$ é um vetor de multiplicadores de Lagrange e $\lambda \in \mathbb{C}$ é um multiplicador de Lagrange escalar. Igualando a zero todas as suas derivadas, obtemos:

$$
\begin{aligned}
& \frac{\dot{o} \mathcal{L}(\tilde{\mathbf{x}}, \mathbf{w}, \mathbf{l}, \lambda)}{\dot{o} \mathbf{l}}=0 \Rightarrow \tilde{\mathbf{X}} \mathbf{w}=0 \\
& \frac{\dot{o} \mathcal{L}(\widetilde{\mathbf{x}}, \mathbf{w}, \mathbf{l}, \lambda)}{\dot{o} \mathbf{w}}=0 \Rightarrow \tilde{\mathbf{X}}^{H} \mathbf{l}=\mathbf{w} \lambda \\
& \frac{\dot{o} \mathcal{L}(\widetilde{\mathbf{x}}, \mathbf{w}, \mathbf{l}, \lambda)}{\dot{o} \lambda}=0 \Rightarrow \mathbf{w}^{H} \mathbf{w}=1 \\
& \frac{\dot{o} \mathcal{L}(\widetilde{\mathbf{x}}, \mathbf{w}, \mathbf{l}, \lambda)}{\partial \tilde{x}_{i}}=0 \Rightarrow\left(x_{i}-\tilde{x}_{i}\right)=\left(\mathbf{l}^{H} \mathbf{X}_{i} \mathbf{w}\right)^{*}
\end{aligned}
$$

para $i=0, \ldots ., N-1$. A partir de $\mathbf{l}^{H} \tilde{\mathbf{X}} \mathbf{w}=\mathbf{0}$ concluímos diretamente que $\lambda=0$. Também, de (81) temos que:

$$
(\mathbf{X}-\tilde{\mathbf{X}}) \mathbf{w}=\sum_{i=0}^{N-1}\left(x_{i}-\tilde{x}_{i}\right) \mathbf{X}_{i} \mathbf{w}=\sum_{i=0}^{N-1}\left(\mathrm{l}^{H} \mathbf{X}_{i} \mathbf{w}\right)^{*} \mathbf{X}_{i} \mathbf{w}
$$

e, de (78), concluímos que:

$$
\begin{aligned}
\mathbf{X w} & =\sum_{i=0}^{N-1}\left(\mathbf{l}^{H} \mathbf{X}_{i} \mathbf{w}\right) \mathbf{X}_{i} \mathbf{w}=\sum_{i=0}^{N-1}\left\{\left[\left(\mathbf{l}^{H} \mathbf{X}_{i} \mathbf{w}\right)^{*} \mathbf{X}_{i} \mathbf{w}\right]^{H}\right\}^{H} \\
& =\sum_{i=0}^{N-1}\left(\mathbf{X}_{i} \mathbf{w}\right)\left(\mathbf{X}_{i} \mathbf{w}\right)^{H} \mathbf{l} \\
& =\mathbf{D}_{w} \mathbf{l}
\end{aligned}
$$

Similarmente, podemos encontrar:

$$
\begin{aligned}
\mathbf{X}^{H} \mathbf{l} & =\sum_{i=0}^{N-1}\left(\mathbf{X}_{i}^{H} \mathbf{l}\right)\left(\mathbf{X}_{i}^{H} \mathbf{l}\right)^{H} \mathbf{w} \\
& =\mathbf{D}_{l} \mathbf{w}
\end{aligned}
$$

Assim, definindo $\mathbf{z}=\mathbf{l} /\|\mathbf{l}\|$ e $\tau=\|\mathbf{l}\|$, escrevemos as seguintes relações:

$$
\begin{array}{lll}
\mathbf{X} \mathbf{w}=\mathbf{D}_{w} \mathbf{z} \tau & \text { tal que } & \mathbf{z}^{H} \mathbf{z}=1 \\
\mathbf{X}^{H} \mathbf{z}=\mathbf{D}_{\mathbf{z}} \mathbf{w} \tau & \text { tal que } & \mathbf{w}^{H} \mathbf{w}=1
\end{array}
$$

Definindo $\quad \mathbf{z}=\mathbf{u} /\|\mathbf{u}\|, \quad \mathbf{w}=\mathbf{v} /\|\mathbf{v}\| \quad$ e $\quad \tau=\sigma\|\mathbf{u}\|\|\mathbf{v}\|$, encontramos:

$$
\begin{array}{lll}
\mathbf{X} \mathbf{v}=\mathbf{D}_{v} \mathbf{u} \sigma & \text { tal que } & \mathbf{u}^{H} \mathbf{D}_{v} \mathbf{u}=1 \\
\mathbf{X}^{H} \mathbf{u}=\mathbf{D}_{u} \mathbf{v} \sigma & \text { tal que } & \mathbf{v}^{H} \mathbf{D}_{u} \mathbf{v}=1
\end{array}
$$

$\operatorname{para} \mathbf{D}_{u} \mathbf{v}=\sum_{i=0}^{N-1} \mathbf{X}_{i}^{H}\left(\mathbf{u}^{H} \mathbf{X}_{i} \mathbf{v}\right) \mathbf{u}$ e $\mathbf{D}_{\nu} \mathbf{u}=\sum_{i=0}^{N-1} \mathbf{X}_{i}\left(\mathbf{u}^{H} \mathbf{X}_{i} \mathbf{v}\right) \mathbf{v} . \quad \mathrm{A}$ relação (86) é conhecida como "SVD Riemanniana" [9].

A partir de (83), podemos escrever:

$$
\mathrm{l}=\mathrm{D}_{w}^{-1} \mathrm{Xw}
$$

Agora, utilizando (81), (83) e (87) podemos escrever a função objetivo do método STLS como:

$$
\begin{aligned}
\sum_{i=0}^{N-1}\left(x_{i}-\tilde{x}_{i}\right)^{H}\left(x_{i}-\tilde{x}_{i}\right) & =\mathbf{l}^{H} \sum_{i=0}^{N-1}\left(\mathbf{l}^{H} \mathbf{X}_{i} \mathbf{w}\right)^{*} \mathbf{X}_{i} \mathbf{w} \\
& =\mathbf{l}^{H} \mathbf{D}_{w} \mathbf{l} \\
& =\mathbf{w}^{H} \mathbf{X}^{H} \mathbf{D}_{w}^{-1} \mathbf{X} \mathbf{w}
\end{aligned}
$$

Assim, de (68) e (81), obtemos:

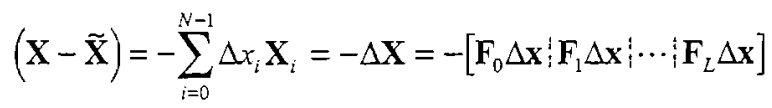

ou seja,

$$
\left[\mathrm{F}_{0} \Delta \mathbf{x}: \mathbf{F}_{t} \Delta \mathbf{x}: \ldots: \mathbf{F}_{L} \Delta \mathbf{x}\right]=\sum_{i=0}^{N-1} \Delta x_{i} \mathbf{X}_{i}
$$

Então, se representarmos a $k$-ésima coluna de $\mathbf{X}_{i}$ por $\mathbf{X}_{i}$ (:, $k)$, para $k=0, \ldots, L$, podemos escrever: 


$$
\begin{aligned}
\mathbf{F}_{k} \Delta \mathbf{x} & =\sum_{i=0}^{N-1} \Delta x_{i} \mathbf{X}_{i}(:, k) \\
& =\left[\mathbf{X}_{0}(:, k): \mathbf{x}_{1}(:, k) \cdots \mathbf{X}_{(N-1)}(:, k)\right] \Delta \mathbf{x}
\end{aligned}
$$

Logo, para qualquer $\Delta \mathbf{x}$, temos que:

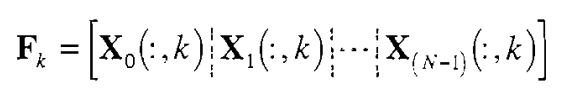

Agora, de (83), podemos escrever $\mathbf{D}_{w}$ como:

$$
\begin{aligned}
\mathbf{D}_{w} & =\sum_{i=0}^{N-1} \mathbf{X}_{i} \mathbf{w}\left(\mathbf{X}_{i} \mathbf{w}\right)^{H} \\
& =\left[\begin{array}{ll:l}
\mathbf{X}_{0} \mathbf{w} & \cdots & \mathbf{X}_{(N-1)} \mathbf{w}
\end{array}\right]\left[\mathbf{X}_{0} \mathbf{w}: \cdots: \mathbf{X}_{(N-1)} \mathbf{w}\right]^{H}
\end{aligned}
$$

porém, utilizando (92) e $\mathbf{W}^{H}=\sum_{i=0}^{L} \mathbf{F}_{i} w_{i}$, encontramos:

$$
\begin{aligned}
& {\left[\begin{array}{l:l:l}
\mathbf{X}_{0} \mathbf{w} & \cdots & \mathbf{X}_{(N-1)} \mathbf{w}
\end{array}\right]=\left[\begin{array}{ll:l:}
\sum_{k=0}^{L} \mathbf{X}_{0}(:, k) w_{k} & \cdots & \sum_{k=0}^{L} \mathbf{X}_{(N-1)}(: k) w_{k}
\end{array}\right]} \\
& =\sum_{k=0}^{L}\left[\mathbf{x}_{0}(:, k): \cdots: \mathbf{x}_{(N-1)}(:, k)\right] v_{k} \\
& =\sum_{k=0}^{L} \mathbf{F}_{k} w_{k} \\
& =\mathrm{W}^{H}
\end{aligned}
$$

Logo, segue-se que:

$$
\mathbf{D}_{w}=\mathbf{W}^{H} \mathbf{W}
$$

Assim, substituindo (95) em (88), finalmente escrevemos a funçāo objetivo do método STLS como:

$$
\min _{\tilde{\mathbf{x}}} \sum_{i=0}^{N-1}\left(x_{i}-\tilde{x}_{i}\right)^{*}\left(x_{i}-\tilde{x}_{i}\right)=\min _{\mathbf{w}}\left\{\mathbf{w}^{H} \mathbf{X}^{H}\left(\mathbf{W}^{H} \mathbf{W}\right)^{-1} \mathbf{X w}\right\}
$$

Novamente, (96) é igual a (73) e (45) e, portanto, o método STLS minimiza o mesmo erro e produz a mesma solução ML que obtemos com os métodos CTLS e IQML.

\section{MÉTODO MÍNIMOS QUADRADOS TOTAIS BRANQUEADO - WTLS}

Devido ao rúdo, em geral não temos uma igualdade em (66), levando a um erro de predição dado por:

$$
\mathbf{X w}=\mathbf{e}
$$

A partir de (44), podemos escrever (97) como:

$$
\mathbf{W}^{H} \mathbf{x}=\mathbf{e}
$$

Outra melhoria à abordagem TLS surge da observação de que este vetor erro não é branco [7]:

$$
\begin{aligned}
E\left\{\mathrm{ee}^{H}\right\} & =E\left\{\mathbf{W}^{H} \mathbf{x x}^{H} \mathbf{W}\right\} \\
& =\mathbf{W}^{H} E\left\{\mathbf{x x}^{H}\right\} \mathbf{W}
\end{aligned}
$$

Substituindo (31) em (99), obtemos:

$$
\begin{aligned}
E\left\{\mathrm{ee}^{H}\right\} & =E\left\{\mathbf{W}^{H}\left(\mathbf{E} \mathbf{a}_{c}+\mathbf{y}\right)\left(\mathbf{E} \mathbf{a}_{c}+\mathbf{y}\right)^{H} \mathbf{W}\right\} \\
& =\mathbf{W}^{H} \mathbf{E a}_{c} \mathbf{a}_{c}^{H} \mathbf{E}^{H} \mathbf{W}+\mathbf{W}^{H} E\left\{\mathbf{y} \mathbf{y}^{H}\right\} \mathbf{W}
\end{aligned}
$$

Entretanto, utilizando (40), podemos escrever (100) como:

$$
\begin{aligned}
E\left\{\mathrm{ee}^{H}\right\} & =\mathbf{W}^{H} E\left\{\mathbf{y} \mathbf{y}^{H}\right\} \mathbf{W} \\
& =\sigma_{y}^{2} \mathbf{W}^{H} \mathbf{W}
\end{aligned}
$$

pois y é branco de variância $\sigma_{y}^{2}$.

Como o vetor erro de predição ótimo é necessariamente branco, o erro TLS jamais será ótimo a menos que ele seja branqueado. Assim, podemos branquear 0 vetor $\mathbf{e}$ simplesmente multiplicando-o por $\left(\mathbf{W}^{H} \mathbf{W}\right)^{-\mathrm{i} / 2}$ para obter:

$$
\left(\mathbf{W}^{H} \mathbf{W}\right)^{-1 / 2} \mathbf{W}^{H} \mathbf{x}=\mathbf{e}_{w}
$$

ou seja,

$$
\left(\mathbf{W}^{H} \mathbf{W}\right)^{-1 / 2} \mathbf{X w}=\mathbf{e}_{\mathbf{w}}
$$

Conseqüentemente, minimizar a norma de $\mathbf{e}_{w}$ corresponde a:

$$
\min \left(\mathbf{e}_{w}^{H} \mathbf{e}_{w}\right)=\min _{\mathbf{w}}\left\{\mathbf{w}^{H} \mathbf{X}^{H}\left(\mathbf{W}^{H} \mathbf{W}\right)^{-1} \mathbf{X} \mathbf{w}\right\}
$$

Outra vez, (104) é igual a (96), (73) e (45). Portanto. concluímos que os métodos WTLS, STLS, CTLS e IQMI minimizam o mesmo erro e produzem a mesma solução ML.

\section{RESULTADOS EXPERIMIEN- TAIS}

No sentido de avaliar o desempenho destes métodos para a estimação de freqüências, escolhemos o mesmo caso utilizado anteriormente. A Fig. 10 apresenta os seguintes desempenhos: da Máxima Verossimilhança teórica (ML, linha pontilhada), do IQML (para $L=2$, linha contínua grossa), do FBLP Modificado (para $L=17$, linha contínua fina), do TLS-FBLP ( para $L=16$, linha tracejada) e o limite de desempenho de Cramér-Rao. Recordamos que os métodos CTLS, WTLS e STLS são equivalentes ao IQML. A convergência ocorreu normalmente em não mais que 20 iterações, sendo em média 5 iteraçōes para SNR de $30 \mathrm{~dB}, 9$ para $10 \mathrm{~dB}$ e 19 para $3 \mathrm{~dB}$.

Podemos verificar que os métodos de predição linear nãoiterativos, como o FBLP Modificado e o TLS-FBLP, 


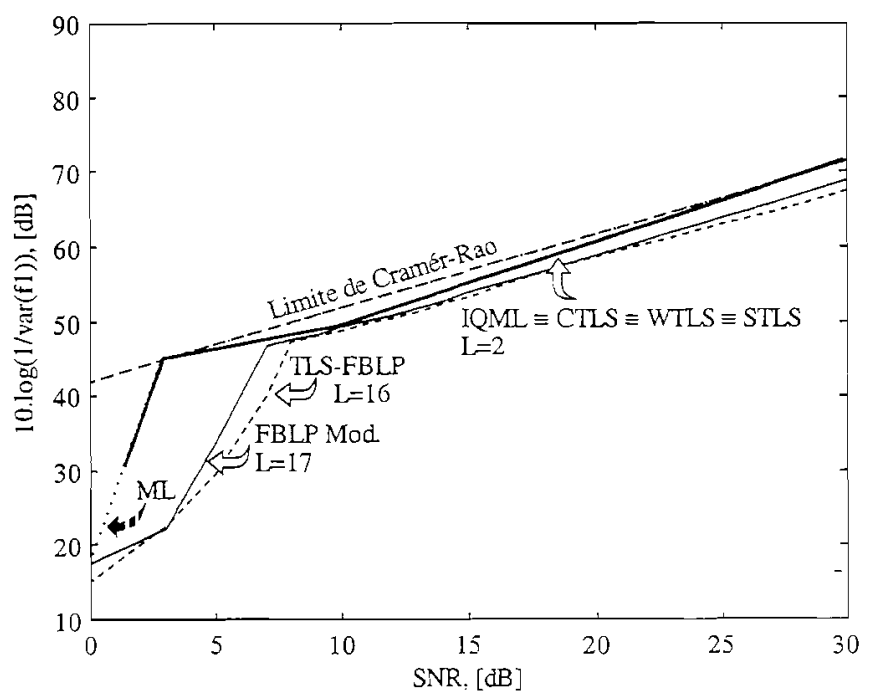

Figura 10. Desempenhos dos métodos estudados e suas respectivas ordens de predição.

estimam satisfatoriamente as freqüências somente em SNRs superiores a $7 \mathrm{~dB}$ e $8 \mathrm{~dB}$, respectivamente. Porém, os métodos de predição linear iterativos, que levam em conta a estrutura da matriz de dados, apresentaram desempenho satisfatório até $\mathrm{SNR}=3 \mathrm{~dB}$, aproximando o desempenho da Máxima Verossimilhança teórica.

Portanto, podemos afirmar que já dispomos de métodos baseados em predição linear que permitem alcançar o desempenho da Máxima Verossimilhança. Estes métodos ¿̃o iterativos e. portanto, apresentam maior complexidade anmutacional do que os métodos FBLP Modificado e TLSFBLP. Porém, ainda assim o esforço computacional exigido pelos métodos iterativos é significativamente inferior àquele da Máxima Verossimilhança.

\section{CONCLUSÕES}

O critério de Mínimos Quadrados Totais (TLS) pode ser aplicado à predição linear forward-backward (FBLP), admitindo erros não somente no vetor desejado $b$, como efetuado por Mínimos Quadrados convencional (LS), mas também na matriz de dados $\mathbf{A}$ e otimizando o vetor $\mathbf{w}$ de coeficientes de predição através de um sistema homogêneo de equações, como no método de Pisarenko.

Isto nos permitiu introduzir o método TLS-FBLP para a estimação de freqüências, o qual se mostrou competitivo com o método FBLP Modificado de Tufts e Kumaresan e menos sensível à variação da ordem de predição, superando o desempenho do FBLP Modificado para ordens pequenas.

Demonstramos analiticamente que os métodos de predição linear iterativos STLS, CTLS, WTLS e IQML minimizam o mesmo erro e produzem soluções equivalentes. Mostramos experimentalmente que o desempenho desses métodos aproxima o da Máxima Verossimilhança para a estimação de frequiências. Portanto, podemos afirmar que já dispomos de métodos baseados em predição linear que permitem alcançar o desempenho da Máxima Verossimilhança.

\section{REFERÊNCIAS}

[1] Krim, H. e Viberg, M., "Two decades of array signc. processing methods", IEEE Signal Processin? Magazine, v. 13, $\mathrm{n}^{\circ}$ 4, pp. 62-94, 1996.

[2] Marple, S.L., Jr., Digital Spectral Analysi: Englewood Cliffs, NJ: Prentice-Hall, 1987.

[3] Tufts, D. W. e Kumaresan, R. "Estimation :-" frequencies of multiple sinusoids: Making lir prediction perform like maximum likeliho: $:$ Proceedings of the IEEE, v. $70, \mathrm{n}^{\circ} 9$, pp. $975-$ 1982.

[4] Golub, G. e Van Loan, C. F. "An analysis of the ::-.. least squares problem", SIAM J. on Numeri:Analysis, v. 17, nº 6, pp. 883-893, 1980.

[5] Lemos, R.P. e Lopes, A. "Frequency detection. linear prediction using total least squares criter: $: . . "$ Proceedings of the $38^{\text {th }}$ Midwest Symposium? Circuits and Systems, v. 2, pp. 823-826, 1995.

[6] Lemos, R.P. e Lopes, A. "Details on the equivalencs of the Modified FBLP method and the TLS-FBLP method", Proceedings of the $39^{\text {th }}$ Midwest Symposium on Circuits and Systems, v. 2, pp. 685-688, 1996.

[7] Hua, Y. e Sarkar, T.K. "On the total least squares linear prediction method for harmonic retrieval problems", IEEE Trans. on Acoustics, Speech and Signal Processing, v. 38, $\mathrm{n}^{\circ}-12$, pp. 2186-2189, 1990.

[8] Abatzoglou, T.J., Mendel, J.M. e Harada, G. "The constrained total least squares technique and its application to harmonic superresolution", IEEE Trans. on Signal Processing, v. 39, $\mathrm{n}^{\circ}$ 5, pp. 1070-1086, 1991 .

[9] De Moor, B. "Total least squares for affinely structured matrices and the noisy realization problem", IEEE Trans, on Signal Processing, v. 42, $\mathrm{n}^{\circ} 11$, pp. 3104-3113, 1994.

[10] Bresler, Y. e Macovski, A. "Exact maximum likelihood estimation of exponential signals in noise", IEEE Trans. on Acoustics, Speech and Signal Processing, v. 34, $\mathrm{n}^{\text {O }}$ 5, pp. 10-1081-1089, 1986.

[11] Lemos, R.P. e Lopes, A. "An unifying framework to total least squares and approximate maximum likelihood", Proceedings of the 1996 Bi-Annual International Telecommunications Symposium (ITS'96), pp. 153-157, 1996.

[12] Ulrych T. J. e R. W. Clayton. "Time series modeling and maximum entropy". Phys. Earth Planet. Interior, v. 12 , pp. $188-200,1976$ 
[13] Nuttal A. H., "Spectral analysis of an univariate process with bad data points via maximum entropy and linear predictive techniques", NUSC Scientific and Engineering Studies, Spectral Estimation, 1976.

[14] Antunes E. J. B. e A. Lopes. "Frequencies estimation of real sinusoids", Proceedings of the International Symposium on Signals, Systems and Electronics ISSSE'92, 1992.

[15] Kay, S.M., Fundamentals of Statiscal Signal Processing: Estimation Theory, Englewood Cliffs, NJ: Prentice-Hall, 1993.

[16] Clark, M.P. e Scharf, L.L. "On the complexity of IQML algorithms", IEEE Trans. on Signal Processing, v. 40, $n^{\mathrm{Q}} 7$, pp. 1811-1813, 1992.

[17] Lemmerling, P., De Moor, B. e Van Huffel, S., "On the equivalence of constrained total least squares and structured least squares", IEEE Trans. on Signal Processing, v. 44, $\mathrm{n}^{\circ}$ 11, pp. 2908-2911, 1996.

[18] Lawson C.L. e R. J. Hanson. Solving Least Squares Problems. Englewood Cliffs: Prentice Hall, 1974.

Amauri Lopes formou-se em Engenharia Elétrica pela Universidade Estadual de Campinas (UNICAMP) em 1972 e obteve o título de mestre e doutor em Engenharia Elétrica pela mesma Universidade em 1974 e 1982, respectivamente. Desde 1973 é professor da Faculdade de Engenharia Elétrica e de Computação da UNICAMP. Atualmente ocupa a posição de Professor Adjunto MS-5. Seus interesses incluem Processamento Digital de Sinais e Comunicações Digitais.

Rodrigo Pinto Lemos graduou-se em Engenharia Elétrica com ênfase em Telecomunicações pela Universidade Federal de Goiás (UFG) em 1992, obtendo os títulos de mestre e doutor em Engenharia Elétrica pela Universidade Estadual de Campinas (UNICAMP) em 1995 e 1997, respectivamente. Atualmente ocupa a posição de Professor Adjunto na Escola de Engenharia Elétrica da UFG, onde se encontra desde 1996. Seus interesses incluem Processamento de Sinais e Redes de Alta Velocidade. 